\title{
Avaliação dos efeitos da adição de sal e da densidade no transporte de tambaqui(1)
}

\author{
Levy de Carvalho Gomes ${ }^{(2)}$, Carlos Alberto Rego Monteiro Araujo-Lima ${ }^{(3)}$, Rodrigo Roubach ${ }^{(3)}$ \\ e Elisabeth Criscuolo Urbinati ${ }^{(4)}$
}

\begin{abstract}
Resumo - Os objetivos deste trabalho foram testar a eficiência do sal como redutor de estresse e verificar a melhor densidade de transporte de juvenis de tambaqui (Colossoma macropomun) em caixas de plástico adaptadas. No primeiro experimento foram testadas diferentes concentrações de sal de cozinha $(\mathrm{NaCl})$ na água; no segundo, o transporte foi realizado por três horas em caixas de plástico de $200 \mathrm{~L}$ estocadas com diferentes densidades de peixe, com $8 \mathrm{~g}$ de sal/L de água. O cortisol plasmático dos peixes sofreu aumento significativo após o transporte no tratamento sem sal e com $2 \mathrm{~g}$ de sal/L de água, retornando para níveis normais após 96 horas. A glicose plasmática dos peixes sofreu aumento após o transporte em todas as concentrações de sal testadas, com exceção da com $8 \mathrm{~g} / \mathrm{L}$ de água, retornando para níveis normais em 24 horas. Nos peixes transportados no segundo experimento, com $8 \mathrm{~g} \mathrm{de} \mathrm{sal/L}$ de água, não foi verificada mudança significativa no cortisol plasmático, mas a glicose aumentou significativamente em todas as densidades após o transporte, retornando para níveis normais em 24 horas. Houve mortalidade de $11 \%$ em uma das repetições da densidade de $200 \mathrm{~kg} / \mathrm{m}^{3}$ de água. Para o transporte com $8 \mathrm{~g}$ de sal/L de água, a densidade máxima deve ser de $150 \mathrm{~kg} / \mathrm{m}^{3}$ de água. Nesta densidade os parâmetros físico-químicos de qualidade de água se mantêm com características adequadas, as respostas ao estresse são mínimas e não há mortalidade.
\end{abstract}

Termos para indexação: Colossoma macropomum, glicose, cloreto de sódio, cortisol, piscicultura.

\section{Assessment on the effect of salt and density on tambaqui fish transportation}

\begin{abstract}
The objectives of this study were to evaluate the efficiency of salt as a stress reductor and to determine the best transportation density for tambaqui (Colossoma macropomum) juveniles in customized plastic boxes. In the first experiment different concentrations of cooking salt $(\mathrm{NaCl})$ in the water were tested, and in the second experiment the fishes were transported for three hours in $200 \mathrm{~L}$ plastic boxes using different fish densities and $8 \mathrm{~g}$ of salt/L of water. Plasma cortisol presented a significant increase after transportation in water without salt or with $2 \mathrm{~g}$ of salt/L, returning to normal levels after 96 hours. The fishes exposed to all salt concentrations had plasma glucose increased after transportation, except the treatment with $8 \mathrm{~g}$ of salt/L of water, returning to normal levels within 24 hours. In the second experiment, the fishes transported at different densities with $8 \mathrm{~g}$ of salt/L of water did not present a significant change in plasma cortisol after transportation, but plasma glucose increased after transportation at all fish densities, returning to normal levels in 24 hours. Fish transported at a density of $200 \mathrm{~kg} / \mathrm{m}^{3}$ of water had $11 \%$ mortality in one of the replicates. For tambaqui transportation with $8 \mathrm{~g}$ of salt $/ \mathrm{L}$ of water, maximum density should be $150 \mathrm{~kg} / \mathrm{m}^{3}$ of water. At this density water parameter levels are adequate, stress responses are minimum and there is no fish mortality.
\end{abstract}

Index terms: Colossoma macropomum, glucose, sodium chloride, cortisol, fish culture.

(1)Aceito para publicação em 31 de outubro de 2002.

Extraído da tese de doutorado apresentada pelo primeiro autor ao Instituto Nacional de Pesquisas da Amazônia (Inpa), Manaus, AM. Financiado pelo Projeto Tambaqui PPD 1139/99, contribuição \# 6 .

(2) Embrapa-Centro de Pesquisa Agroflorestal da Amazônia Ocidental, Caixa Postal 319, CEP 69011-970 Manaus, AM. E-mail: levy@cpaa.embrapa.br

(3) Inpa, Caixa Postal 478, CEP 69011-970 Manaus, AM E-mail: calima@inpa.gov.br, roubach@inpa.gov.br

(4) Universidade Estadual Paulista, Centro de Aqüicultura, Rod Paulo Donato Castellane, km 5, CEP 14870-000 Jaboticabal, SP. E-mail: bethurb@caunesp.unesp.br

\section{Introdução}

O transporte é uma prática rotineira em sistemas de criação de peixes (Piper et al., 1982; Carmichel et al., 2001). No norte do Brasil, o transporte de juvenis (1-2 kg) vem aumentando consideravelmente e as principais finalidades são o comércio de peixe vivo em feiras para consumo, fornecimento para fazendas e sítios de pesque-pague e formação de plantel de reprodutores. 
Na Amazônia, o transporte de peixes com tamanho para o abate é feito no sistema aberto, com fornecimento constante de oxigênio. A obtenção de caixas confeccionadas especialmente para o transporte é difícil na região, além do custo e do frete serem proibitivos para a maioria dos produtores. Desta forma, as caixas mais utilizadas são galões de plástico, cilíndricos, com capacidade para 200 L e confeccionados originalmente para o transporte de produtos em conserva. Esses galões também são utilizados para o transporte de peixes em outras partes do mundo (Berka, 1986).

Durante o transporte, os peixes são afetados por uma série de agentes estressantes: captura, confinamento, manuseio e o próprio transporte. A quantificação do estresse ao qual o peixe é submetido durante o transporte é fundamental para que se estabeleçam práticas de manejo adequadas (Wedemeyer, 1997). As respostas ao estresse são divididas em três categorias: primária, secundária e terciária (Mazeaud et al., 1977; Wedemeyer \& McLeay, 1981). As respostas primárias são as hormonais, as secundárias são as mudanças nos parâmetros fisiológicos e bioquímicos; as terciárias são o comprometimento do crescimento, mudanças no comportamento e aumento da suscetibilidade a doenças. Os indicadores mais utilizados na avaliação do estresse e que normalmente dão uma boa resposta são a glicose e o cortisol plasmáticos (Robertson et al., 1987; Barton, 2000). O cortisol é utilizado para caracterizar a resposta primária, e a glicose, a resposta secundária.

O uso do sal de cozinha como redutor de estresse é amplamente difundido na aqüicultura para igualar o gradiente osmótico entre a água e o plasma do peixe, fazendo com que haja uma redução na difusão de íons para a água. O sal também estimula a secreção de muco sobre o epitélio branquial, dificultando a passagem de íons através das membranas celulares (Wurts, 1995). Além de reduzir o estresse, o sal também tem efeito profilático, é de fácil obtenção e baixo custo, com eficácia comprovada em várias espécies (Barton \& Zitzow, 1995; Allyn et al., 2001). Por causa dessas propriedades, o sal é normalmente utilizado durante o transporte de peixes. No entanto, os produtores o usam de forma empírica, ou seja, sem conhecimento da concentração ideal.

O tambaqui (Colossoma macropomum) é o peixe mais criado na Região Amazônica (Val et al., 2000), principalmente pela fácil obtenção de juvenis, bom potencial de crescimento, alta produtividade e rusticidade (Araujo-Lima \& Goulding, 1997). É muito apre- ciado pela população local e a demanda por sua carne é grande, razão pela qual muitos pesquisadores e produtores têm intensificado esforços para estabelecer um pacote tecnológico para a criação da espécie. Apesar disso, pouca atenção foi dada ao transporte (Araujo-Lima \& Goulding, 1997).

Segundo Grottum et al. (1997) e Wedemeyer (1997), o principal fator de sucesso do transporte é conter a maior densidade de peixes no menor volume de água possível, sem que haja mortalidade, deterioração da qualidade da água e estresse.

Os objetivos deste trabalho foram testar a eficiência do sal como redutor do estresse e verificar a melhor densidade de transporte de juvenis de tambaqui em caixas de plástico adaptadas.

\section{Material e Métodos}

Juvenis de tambaqui foram estocados com cerca de $20 \mathrm{~g}$ em um viveiro de $500 \mathrm{~m}^{2}$ na piscicultura e pesque-pague San Diego, em Manaus, AM. O viveiro era abastecido por água de poço artesiano, com aeradores mecânicos ligados em dias em que a concentração de oxigênio na água era inferior a 3,0 mg/L. Os peixes receberam ração comercial quatro vezes por semana, durante um ano, até atingirem peso médio de $846 \pm 25 \mathrm{~g}$ (média \pm erro-padrão).

Os transportes foram realizados entre janeiro e fevereiro de 2002, sempre entre $7 \mathrm{~h}$ e $11 \mathrm{~h}$. Antes do transporte, os peixes ficaram sem alimentação por 48 horas para depuração gastrintestinal (Grottum et al., 1997). O transporte foi realizado em três caixas de plástico cilíndricas, com capacidade para $200 \mathrm{~L}$ cada, com a face externa pintada de branco, confeccionadas originalmente para o transporte de produtos em conserva e adaptadas para o transporte de peixes. Durante o transporte, as caixas receberam oxigênio por um cilindro acoplado a um manômetro. As concentrações de oxigênio foram monitoradas a cada 30 minutos e mantidas entre 4,0 e $8,0 \mathrm{mg} / \mathrm{L}$. Os peixes foram transportados por rodovia durante três horas e levados para três viveiros de $25 \mathrm{~m}^{2}$, localizados na Coordenação de Pesquisas em Aquacultura do Instituto Nacional de Pesquisas da Amazônia. Os viveiros eram abastecidos por água de poço artesiano e equipados com sopradores de ar. A concentração de oxigênio na água dos viveiros foi mantida sempre acima de 3,0 $\mathrm{mg} / \mathrm{L}$ por meio de troca periódica da água (diariamente durante 12 horas) e de uso do soprador. Os peixes permaneceram nos viveiros por 96 horas, e durante esse período foi fornecida ração comercial diariamente às 7 h30 e 16 horas. O consumo de ração foi avaliado por observação visual. Também foram avaliadas as respostas fisiológicas e a mortalidade após o transporte. 
No primeiro experimento, os peixes foram transportados na densidade de $65 \mathrm{~kg} / \mathrm{m}^{3}$ de água (15 peixes) em águas que continham sal de cozinha nas concentrações de $0,2,5$ e $8 \mathrm{~g} / \mathrm{L}$ de água. Essas concentrações foram escolhidas por estarem dentro da faixa recomendada pela literatura especializada (Ross \& Ross, 1999). Foram realizadas quatro viagens, em cada uma havia três caixas com diferentes concentrações de sal, de modo que cada concentração foi repetida três vezes.

No segundo experimento, foram testadas as densidades de 100,150 e $200 \mathrm{~kg} / \mathrm{m}^{3}$ de água contendo 23,35 e 46 peixes, respectivamente. A concentração de sal usada foi de $8 \mathrm{~g} / \mathrm{L}$ de água por ter sido a mais eficiente no primeiro experimento. Foram realizadas duas viagens, sendo cada densidade repetida duas vezes.

A qualidade da água foi avaliada nos seguintes momentos do transporte: 0 minuto, 90 minutos e 180 minutos. Oxigênio e temperatura foram medidos com oxímetro YSI 55, pH com peagômetro digital, dióxido de carbono por titulação de acordo com a American Public Health Association (1992). A amônia total $\left(\mathrm{NH}_{3}+\mathrm{NH}_{4}\right)$ foi medida segundo Verdow et al. (1978) e as concentrações da amônia não ionizada $\left(\mathrm{NH}_{3}\right)$ foram calculadas de acordo com Boyd (1982).

Foi coletado sangue de quatro peixes de cada repetição na captura do viveiro (antes do transporte; AT), imediatamente após o transporte (PT) e 24 horas e 96 horas após o transporte (24PT e 96PT). Na coleta, os peixes foram rapidamente capturados e anestesiados com $100 \mathrm{mg}$ de
benzocaína/L de água (Gomes et al., 2001). O sangue foi coletado da veia caudal com a utilização de seringas heparinizadas e transferido para tubos de eppendorf, mantidos em gelo fundente. $\mathrm{O}$ plasma foi então separado por centrifugação a $3.000 \mathrm{~g}$, durante 10 minutos. As concentrações de glicose plasmática foram determinadas com auxílio de um sistema enzimático colorimétrico. Uma alíquota de plasma foi congelada a $-80^{\circ} \mathrm{C}$ para análise de cortisol, pela técnica de radioimunoensaio.

Os dados obtidos foram submetidos à analise de variância e, para os parâmetros de qualidade de água, as médias entre os tratamentos e entre os diferentes momentos de um mesmo tratamento foram comparadas pelo teste de Tukey a 5\% de probabilidade. Em relação aos resultados de estresse, as médias entre os tratamentos e o controle foram comparadas pelo teste de Dunnet a $5 \%$ de probabilidade. Todas as análises foram realizadas no pacote estatístico Systat 9.0.

\section{Resultados e Discussão}

\section{Experimento 1}

A temperatura teve aumento significativo no tempo de 180 minutos dentro de cada tratamento, com exceção do que não recebeu sal (Tabela 1). Esse resultado era esperado, uma vez que o final do transporte ocorria entre $10 \mathrm{~h}$ e $11 \mathrm{~h}$, quando a temperatura ambiental era mais alta $\left(28-30^{\circ} \mathrm{C}\right)$, em comparação à

Tabela 1. Qualidade da água durante o transporte de tambaqui em diferentes concentrações de sal de cozinha na água ${ }^{(1)}$.

\begin{tabular}{|c|c|c|c|c|}
\hline \multirow{2}{*}{$\begin{array}{l}\text { Tempo de transporte } \\
\text { (minuto) }\end{array}$} & \multicolumn{4}{|c|}{ Sal de cozinha (g/L de água) } \\
\hline & 0 & 2 & 5 & 8 \\
\hline & \multicolumn{4}{|c|}{ Temperatura $\left({ }^{\circ} \mathrm{C}\right)$} \\
\hline 0 & $26,9 \pm 0,67 \mathrm{aA}$ & $26,9 \pm 0,06 \mathrm{aA}$ & $26,4 \pm 0,30 \mathrm{aA}$ & $26,8 \pm 0,09 \mathrm{aA}$ \\
\hline 90 & $27,7 \pm 0,10 \mathrm{aA}$ & $27,3 \pm 0,17 \mathrm{aA}$ & $27,4 \pm 0,20 \mathrm{aB}$ & $27,3 \pm 0,12 \mathrm{aA}$ \\
\hline 180 & $28,3 \pm 0,24 \mathrm{aA}$ & $28,1 \pm 0,29 \mathrm{aB}$ & $28,0 \pm 0,15 \mathrm{aB}$ & $28,2 \pm 0,24 \mathrm{aB}$ \\
\hline \multicolumn{5}{|c|}{$\mathrm{pH}$} \\
\hline 0 & $5,02 \pm 0,54 \mathrm{aA}$ & $5,20 \pm 0,46 \mathrm{aA}$ & $5,43 \pm 0,09 \mathrm{aA}$ & $5,22 \pm 0,44 \mathrm{aA}$ \\
\hline 90 & $5,11 \pm 0,25 \mathrm{aA}$ & $5,17 \pm 0,31 \mathrm{aA}$ & $5,51 \pm 0,01 \mathrm{aA}$ & $5,29 \pm 0,29 \mathrm{aA}$ \\
\hline 180 & $5,34 \pm 0,24 \mathrm{aA}$ & $5,21 \pm 0,22 \mathrm{aA}$ & $5,42 \pm 0,09 \mathrm{aA}$ & $5,15 \pm 0,20 \mathrm{aA}$ \\
\hline & \multicolumn{4}{|c|}{$\mathrm{CO}_{2}(\mathrm{mg} / \mathrm{L})$} \\
\hline 0 & $34,47 \pm 7,66 \mathrm{aA}$ & $25,67 \pm 9,19 \mathrm{aA}$ & $35,93 \pm 12,07 \mathrm{aA}$ & $25,67 \pm 11,53 \mathrm{aA}$ \\
\hline 90 & $38,13 \pm 4,81 \mathrm{aA}$ & $33,00 \pm 19,05 \mathrm{aA}$ & $28,60 \pm 6,60 \mathrm{aA}$ & $24,20 \pm 4,58 \mathrm{aA}$ \\
\hline 180 & $41,80 \pm 7,07 \mathrm{aA}$ & $35,20 \pm 6,72 \mathrm{aA}$ & $38,13 \pm 10,65 \mathrm{aA}$ & $33,00 \pm 5,54 \mathrm{aA}$ \\
\hline & \multicolumn{4}{|c|}{$\mathrm{NH}_{3}(\mu \mathrm{mol} / \mathrm{L})$} \\
\hline 0 & $0,03 \pm 0,03 \mathrm{aA}$ & $0,07 \pm 0,03 \mathrm{aA}$ & $0,09 \pm 0,02 \mathrm{aA}$ & $0,09 \pm 0,06 \mathrm{aA}$ \\
\hline 90 & $3,71 \pm 0,58 \mathrm{aB}$ & $2,77 \pm 0,23 \mathrm{aB}$ & $3,54 \pm 0,61 \mathrm{aB}$ & $3,32 \pm 0,79 \mathrm{aB}$ \\
\hline 180 & $5,34 \pm 1,19 \mathrm{aB}$ & $6,30 \pm 1,85 \mathrm{aB}$ & $5,86 \pm 0,84 \mathrm{aB}$ & $5,01 \pm 0,19 \mathrm{aB}$ \\
\hline
\end{tabular}

(1)Médias seguidas pela mesma letra, minúscula na linha e maiúscula na coluna, não diferem entre si pelo teste de Tukey a 5\% de probabilidade; os dados representam as médias ¿erro-padrão de três repetições de cada concentração de sal. 
do início do transporte $\left(26-27^{\circ} \mathrm{C}\right)$, entre $7 \mathrm{~h}$ e $8 \mathrm{~h}$. Os valores de $\mathrm{pH}$ e as concentrações de $\mathrm{CO}_{2}$ não tiveram alteração significativa entre os diferentes tempos em nenhum tratamento. $\mathrm{O} \mathrm{CO}_{2}$ inicial foi alto, por ter sido utilizada água de poço artesiano. Mesmo com a manutenção e movimentação desta água na caixa de transporte, antes do experimento, não foi possível retirar todo $\mathrm{CO}_{2}$ existente. A manutenção dos valores em níveis baixos durante o transporte se deve, principalmente, à baixa densidade de peixes. Além disso, essas concentrações estiveram bem abaixo das consideradas críticas para a maioria dos peixes durante o transporte (Berka, 1986; Grottum et al., 1997). Desta forma, o $\mathrm{CO}_{2}$ não deve ter sido causador de distúrbios durante o transporte.

Houve aumento de $\mathrm{NH}_{3}$ do tempo 0 para 90 minutos, permanecendo com valores iguais a este no tempo 180 minutos (Tabela 1). As águas ácidas, além de serem as mais comuns em estações de piscicultura da Região Amazônica, são uma característica marcante de muitos corpos de água dessa região (Wood et al., 1998). O caráter ácido da água utilizada no experimento fez com que apenas $0,08 \%$ da amônia total $\left(\mathrm{NH}_{3}+\mathrm{NH}_{4}\right)$ estivesse em seu estado tóxico $\left(\mathrm{NH}_{3}\right)$. Desta forma, a amônia teve uma importância secundária durante o transporte de curta distância. Resultado semelhante foi observado por Gomes et al. (1999) durante o transporte de juvenis de jundiá (Rhamdia quelen) em sistema fechado, no sul do Brasil, onde o pH da água (em torno de 7) é mais alto que os da Região Amazônica.

O cortisol plasmático apresentou aumento nos tratamentos com 0 e $2 \mathrm{~g}$ de sal/L de água no momento PT (Figura 1). Na amostragem de 24PT, os valores do cortisol, nos tratamentos com 0,2 e $5 \mathrm{~g}$ de sal/L de água, foram superiores ao do controle. As concentrações de cortisol retornaram para valores iguais aos do controle na amostragem de 96PT em todos os tratamentos. O tratamento com $8 \mathrm{~g}$ de sal/L de água não teve alteração significativa do cortisol em nenhum momento de amostragem. Houve aumento da glicose plasmática nos tratamentos com 0,2 e $5 \mathrm{~g}$ de sal/L de água no momento PT, retornando para valores iguais ao controle no momento 24PT (Figura 1). Os peixes do tratamento com $8 \mathrm{~g}$ de sal/L de água não tiveram alteração na glicose em nenhum momento da amostragem.
O sal de cozinha na maior concentração testada ( $8 \mathrm{~g} / \mathrm{L}$ de água) foi eficiente para suprimir a liberação de cortisol. Resultado semelhante foi obtido para matrinxã (Brycon cephalus) após quatro horas de transporte em uma densidade de $150 \mathrm{~kg} / \mathrm{m}^{3}$, usando $6 \mathrm{~g}$ de sal/L de água (Carneiro \& Urbinati, 2001). A principal causa deve ter sido um ajuste de gradiente entre o peixe e o ambiente, diminuindo a pressão osmótica e o trabalho osmorregulatório (Ross \& Ross, 1999). A concentração de glicose em peixes transportados com $8 \mathrm{~g}$ de sal/L de água reforça o argumento de que a concentração de sal de cozinha utilizada evita gasto energético para o peixe durante o processo de transporte.

Os peixes de todos os tratamentos aceitaram ração na primeira oferta (em torno de 18 horas após o
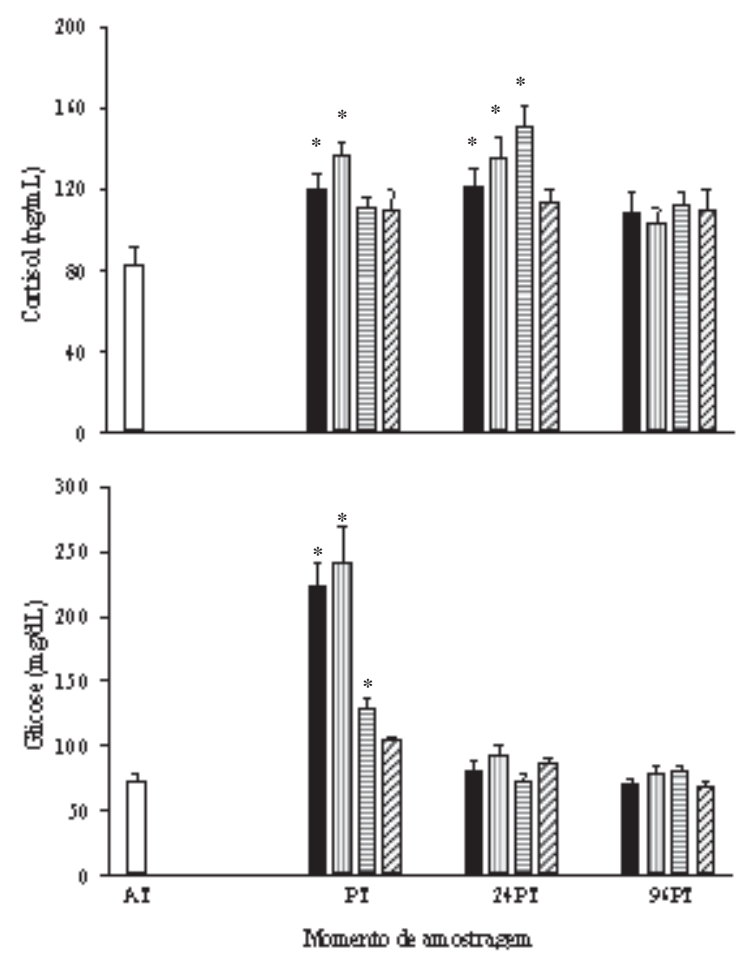

Figura 1. Médias das concentrações de cortisol e glicose plasmáticos durante o transporte de tambaqui, com $0(\boldsymbol{\square})$, 2 (ㅁ), 5 (ㅁ) e 8 (घ) g de sal de cozinha/L de água, antes do transporte (AT), após o transporte (PT), 24 e 96 horas após o transporte (24PT e 96PT). *Significativo em relação ao controle (AT) pelo teste de Dunnett a 5\% de probabilidade ( $\mathrm{n}=4$ para cada repetição). As barras em cada coluna representam o erro-padrão. 
transporte), porém passaram a se alimentar da forma habitual após 48 horas. Não foi observada nenhuma diferença no consumo de ração nem na mortalidade dos peixes dos diferentes tratamentos.

\section{Experimento 2}

A temperatura aumentou no tempo 180 minutos nas densidades de 100 e $150 \mathrm{~kg} / \mathrm{m}^{3}$ de água (Tabela 2). $\mathrm{O} \mathrm{CO}_{2}$ teve aumento do tempo 0 para o tempo 90 minutos na densidade de $100 \mathrm{~kg} / \mathrm{m}^{3} \mathrm{de}$ água e um aumento em todos os tempos de amostragem na densidade $150 \mathrm{~kg} / \mathrm{m}^{3}$ de água. A amônia total aumentou de um tempo para outro em todas as densidades (Tabela 2).

As concentrações de cortisol plasmático não tiveram alteração significativa em nenhuma densidade e em nenhum momento de amostragem (Figura 2). As concentrações de cortisol nas densidades testadas nos diferentes momentos de amostragem (101,41-137,14 ng/mL) foram semelhantes às obtidas no tratamento com $8 \mathrm{~g}$ de sal/L de água do experimento $1(109,45-112,94 \mathrm{ng} / \mathrm{mL})$. Tal resultado evidencia que o sal de cozinha na concentração adequada é eficiente para causar uma supressão da liberação de cortisol.

A glicose plasmática sofreu aumento significativo no momento PT nas três densidades, retornando para valores iguais ao do controle no momento 24PT (Figura 2). O aumento da glicose está normalmente relacionado com a liberação de cortisol e outros hormônios, principalmente catecolaminas (Mommsen et al., 1999). No segundo experimento, o aumento da glicose plasmática no momento PT deve ter sido ocasionado pela liberação das catecolaminas, pois o cortisol plasmático permaneceu inalterado. Como a resposta da glicose e do cortisol apresentaram um padrão diferente para o tambaqui no segundo experimento, não se pode concluir se houve ou não uma situação estressante para os peixes com base apenas em um indicador fisiológico, quando se adiciona o sal de cozinha como redutor de estresse.

O sal de cozinha ( $8 \mathrm{~g} / \mathrm{L}$ de água) foi eficiente para reduzir a resposta da glicose. No primeiro experimento, a concentração de glicose foi $223,34 \pm 18,69 \mathrm{mg} / \mathrm{dL}$ no tratamento sem sal (0 g/L de água), e $103,83 \pm 3,70 \mathrm{mg} / \mathrm{dL}$ no tratamento com $8 \mathrm{~g}$ de sal/L de água no momento PT. No segundo experimento, o valor da glicose nas diferentes densidades foi muito similar e variou de 92,23 a $100,56 \mathrm{mg} / \mathrm{dL}$ no momento PT, valores estes semelhantes ao obtido no primeiro experimento com $8 \mathrm{~g}$ de sal/L de água. Tais valores também são cerca de 2,2 vezes mais baixos que os do tratamento sem sal do primeiro experimento.

Tabela 2. Qualidade da água durante o transporte de tambaqui em diferentes densidades de carga com $8 \mathrm{~g}$ de sal/L de água ${ }^{(1)}$.

\begin{tabular}{cccc}
\hline \multirow{2}{*}{$\begin{array}{c}\text { Tempo de transporte } \\
\text { (minuto) }\end{array}$} & \multicolumn{3}{c}{ Densidade de carga $\left(\mathrm{kg} / \mathrm{m}^{3}\right.$ de água) } \\
\cline { 2 - 4 } 0 & 100 & 150 & 200 \\
\hline & & Temperatura $\left({ }^{\circ} \mathrm{C}\right)$ & \\
90 & $27,85 \pm 0,05 \mathrm{aA}$ & $27,95 \pm 0,05 \mathrm{aA}$ & $26,75 \pm 1,15 \mathrm{aA}$ \\
180 & $28,10 \pm 0,10 \mathrm{aA}$ & $28,15 \pm 0,05 \mathrm{aA}$ & $27,05 \pm 1,05 \mathrm{aA}$ \\
0 & $28,85 \pm 0,05 \mathrm{aB}$ & $28,85 \pm 0,05 \mathrm{aB}$ & $27,6 \pm 0,5 \mathrm{aA}$ \\
\hline & $\mathrm{pH}$ \\
90 & $5,48 \pm 0,17 \mathrm{aA}$ & $5,50 \pm 0,12 \mathrm{aA}$ & $5,43 \pm 0,05 \mathrm{aA}$ \\
180 & $5,34 \pm 0,14 \mathrm{aA}$ & $5,56 \pm 0,10 \mathrm{aA}$ & $5,60 \pm 0,06 \mathrm{aA}$ \\
0 & $5,60 \pm 0,06 \mathrm{aA}$ & $5,81 \pm 0,15 \mathrm{aA}$ & $5,81 \pm 0,15 \mathrm{aA}$ \\
\hline & & $\mathrm{CO} 2(\mathrm{mg} / \mathrm{L})$ \\
180 & $22,0 \pm 0,0 \mathrm{aA}$ & $22,0 \pm 0,0 \mathrm{aA}$ & $17,6 \pm 4,4 \mathrm{aA}$ \\
0 & $31,9 \pm 1,1 \mathrm{aB}$ & $34,1 \pm 1,1 \mathrm{aB}$ & $35,2 \pm 0,0 \mathrm{aA}$ \\
90 & $33,0 \pm 2,2 \mathrm{aB}$ & $42,9 \pm 1,1 \mathrm{aC}$ & $56,1 \pm 12,11 \mathrm{aA}$ \\
180 & & $\mathrm{NH}(\mu \mathrm{mol} / \mathrm{L})$ \\
& $0,11 \pm 0,09 \mathrm{aA}$ & $0,17 \pm 0,14 \mathrm{aA}$ & $0,09 \pm 0,06 \mathrm{aA}$ \\
& $4,66 \pm 0,05 \mathrm{aB}$ & $5,41 \pm 0,96 \mathrm{aB}$ & $6,64 \pm 0,13 \mathrm{aB}$ \\
& $6,84 \pm 0,32 \mathrm{aC}$ & $8,22 \pm 0,93 \mathrm{aB}$ & $10,07 \pm 0,24 \mathrm{aC}$ \\
\hline
\end{tabular}

${ }^{(1)}$ Médias seguidas pela mesma letra, minúscula na linha e maiúscula na coluna, não diferem entre si pelo teste de Tukey a 5\% de probabilidade; os dados representam as médias \pm erro-padrão de duas repetições de cada densidade. 
Nos dois experimentos, a alteração da glicose só ocorreu após o transporte, retornando para níveis normais na amostragem de 24PT, ou seja, o tambaqui recuperou rapidamente sua condição glicêmica normal. Esta rápida recuperação glicêmica em situações estressantes já é conhecida nessa espécie (Wood et al., 1998). Resultado semelhante foi obtido por Robertson et al. (1987) com red drum (Sciaenops ocellatus). Outro caraciforme da Amazônia, o matrinxã, tem uma recuperação mais lenta em situações estressantes de transporte, necessitando de 96 horas (Carneiro \& Urbinati, 2001) ou mais (Urbinati $\&$ Carneiro, 2001) para o retorno dos valores normais de glicose.
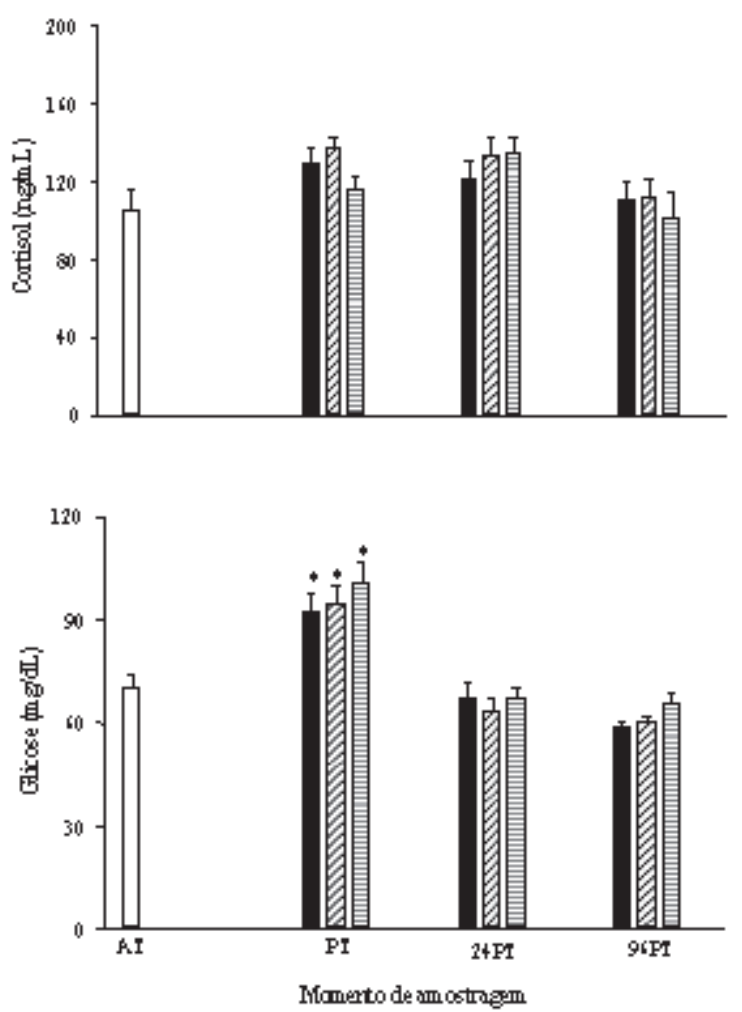

Figura 2. Médias das concentrações de cortisol e glicose plasmáticos durante o transporte de tambaqui com $100(\square), 150$ (ㅁ) e 200 (ㅁ) $\mathrm{kg}$ de peixe/ $\mathrm{m}^{3}$ de água com $8 \mathrm{~g}$ de sal/L de água, antes do transporte (AT), após o transporte (PT), 24 e 96 horas após o transporte (24PT e 96PT). *Significativo em relação ao controle (AT) pelo teste de Dunnett a $5 \%$ de probabilidade ( $\mathrm{n}=4$ para cada repetição). As barras em cada coluna representam o erro-padrão.
Os peixes das duas menores densidades aceitaram ração na primeira oferta ( em torno de 18 horas após o transporte), voltando ao consumo habitual após 48 horas. Por sua vez, os peixes da maior densidade (200 kg/m³ de água) praticamente não aceitaram ração. Apesar de não ter sido quantificado o consumo, era nítido que os peixes da maior densidade se alimentaram menos que os demais. Na densidade de $200 \mathrm{~kg} / \mathrm{m}^{3}$ de água ocorreu uma mortalidade de $11 \%$ (quatro peixes) em uma das repetições, na qual havia também peixes que apresentavam lesões no corpo. $\mathrm{Na}$ outra repetição desta densidade não houve mortalidade, porém também verificaram-se peixes com lesão. Em ambas as repetições, os peixes apresentavam comportamento incomum, natação lenta em direção à superfície nas primeiras 48 horas após o transporte. Após esse período, os peixes voltaram a apresentar comportamento normal, nadando em meia profundidade. Esse resultado é indicativo de que a densidade $200 \mathrm{~kg} / \mathrm{m}^{3}$ de água é extrema para o tambaqui transportado em caixas adaptadas.

A baixa capacidade de carga $\left(150 \mathrm{~kg} / \mathrm{m}^{3}\right.$ de água $)$ é fator limitante para o transporte de grandes quantidades de peixes nas caixas utilizadas no presente trabalho. Essa densidade equivale à metade da recomendada por Kubitza (1998) para o transporte de tambaqui em caixas apropriadas (300 kg/ $\mathrm{m}^{3}$ de água). Mesmo assim, as caixas adaptadas mostraram-se eficientes no transporte de pequenas quantidades de tambaqui.

\section{Conclusões}

1. O sal de cozinha na concentração de $8 \mathrm{~g} / \mathrm{L}$ de água é eficiente para diminuir a maioria das respostas fisiológicas do estresse em tambaquis durante o transporte.

2. No transporte em caixas adaptadas com $8 \mathrm{~g}$ de sal/L de água, a densidade de $150 \mathrm{~kg} / \mathrm{m}^{3}$ de água é a mais adequada.

3. As caixas adaptadas são eficientes para o transporte.

\section{Agradecimentos}

Aos pesquisadores Manoel Pereira Filho, Flávio Fonseca, Bruno Cavero, pela ajuda prestada durante a realização deste trabalho; ao pesquisador Richard Brinn, pela ajuda na análise de cortisol; à Coordenação de Pesquisas em Aquacultura/Cpaq do Inpa, pelo apoio logístico. 


\section{Referências}

ALLYN, M. L.; SHEEHAN, R. J.; KOHLER, C. C. The effects of capture and transportation stress on white bass semen osmolarity and their alleviation via sodium chloride. Transactions of the American Fisheries Society Bethesda, v. 130, n. 4, p. 706-711, 2001.

AMERICAN PUBLIC HEALTH ASSOCIATION (Baltimore, Estados Unidos). Standard methods for the examination of water and wastewater. 18th ed. New York, 1992. $1220 \mathrm{p}$.

ARAUJO-LIMA, C. R. M.; GOULDING, M. So fruitful fish: ecology, conservation, and aquaculture of the Amazon's tambaqui. New York: Columbia University Press, 1997. $157 \mathrm{p}$.

BARTON, B. A. Salmonid fishes differ in their cortisol and glucose responses to handling and transport stress. North American Journal of Aquaculture, Bethesda, v. 62, n. 1 , p. $12-18,2000$.

BARTON, B. A.; ZITZOW, R. E. Physiological responses of juvenile walleyes to handling stress with recovery in saline water. Progressive Fish-Culturist, Bethesda, v. 57, p. 267-276, 1995.

BERKA, R. The transport of live fish: a review. Rome: FAO, 1986. 57 p. (EIFAC Technical Papers, 48).

BOYD, C. E. Water quality management for pond fish culture. Amsterdam: Elsevier Science, 1982. 317 p.

CARMICHEL, G. J.; TOMASSO, J. R.; SCHWEDLER, T. E. Fish transportation. In: WEDEMEYER, G. A. (Ed.). Fish hatchery management. 2nd ed. Bethesda: American Fisheries Society, 2001. p. 641-660.

CARNEIRO, P. C. F.; URBINATI, E. C. Salt as a stress response mitigator of matrinxã, Brycon cephalus (Günther), during transport. Aquaculture Research, Oxford, v. 32, n. 4, p. 298-307, 2001.

GOMES, L. C.; CHIPPARI-GOMES, A. R.; LOPES, N. P.; ROUBACH, R.; ARAUJO-LIMA, C. A. R. M. Efficacy of benzocaine as anesthetic for tambaqui juveniles (Colossoma macropomum). Journal of the World Aquaculture Society, Baton Rouge, v. 31, n. 4, p. 426-431, 2001.

GOMES, L. C.; GOLOMBIESKI, J. I.; CHIPPARI-GOMES, A. R.; BALDISSEROTTO, B. Effect of salt in the water of transport on survival and $\mathrm{Na}^{+}$and $\mathrm{K}^{+}$body levels in fingerlings of silver catfish Rhamdia quelen (Pimelodidae). Journal of Applied Aquaculture, Binghamton, v. 9, n. 4, p. $1-9,1999$.
GROTTUM, J. A.; STAURNES, M.; SIGHOLT, T. Effect of oxygenation, aeration and $\mathrm{pH}$ control on water quality and survival of turbot, Scophthalmus maximus (L.), kept at high densities during transport. Aquaculture Research, Oxford, v. 28, n. 2, p. 159-164, 1997.

KUBITZA, F. Técnicas de transporte de peixes vivos. Campo Grande: Conceito, 1998. 44 p.

MAZEAUD, M. M.; MAZEAUD, F.; DONALDSON, E. M. Primary and secondary effects of stress in fish: some new data with a general review. Transactions of the American Fisheries Society, Binghamton, v. 106, p. 201-212, 1977.

MOMMSEN, T. P; VIJAYAN, M. M; MOON, T. W. Cortisol in teleosts: dynamics, mechanisms of action, and metabolic regulation. Reviews in Fish Biology and Fisheries, Dordrecht, v. 9, n. 3, p. 211-268, 1999.

PIPER, G. R.; McELWAIN, I. B.; ORME, L. E.; McCRAREN, J. P.; FOWLER, L. G.; LEONARD, J. R. Fish hatchery management. Washington: United States Department of the Interior, 1982. $517 \mathrm{p}$.

ROBERTSON, L.; THOMAS, P.; ARNOLD, C. R.; TRANT, J. M. Plasma cortisol and secondary stress responses of red drum to handling, transport, rearing density, and a disease outbreak. Progressive Fish-Culturist, Binghamton, v. 49, n. 1, p. 1-12, 1987.

ROSS, L. G.; ROSS, B. Anaesthetic and sedative techniques for aquatic animals. Oxford: Blackwell Science, 1999. 159 p.

URBINATI, E. C.; CARNEIRO, P. C. F. Metabolic and hormonal responses of matrinxã, Brycon cephalus, (Teleost: Characidae) to transport stress under influence of benzocaine. Journal of Aquaculture in the Tropics, New Delhi, v. 16, n. 1, p. 75-85, 2001.

VAL, A. L.; ROLIM, P. R.; RABELO, H. Situação atual da aqüicultura na Região Norte. In: VALENTE, W. C.; POLI, C. R.; PEREIRA, J. A.; BORGHETTI, J. R. (Ed.). Aquiicultura no Brasil: bases para um desenvolvimento sustentável. Brasília: CNPq/MCT, 2000. p. 247-266.

VERDOW, H.; VANECHTED, C. J. A.; DEKKERS, E. M. J. Ammonia determination based on indophenol with sodium salicylate. Water Research, Amsterdam, v. 12, p. 399-402, 1978.

WEDEMEYER, G. A. Effect of rearing conditions on the health and physiological quality of fish in intensive culture. In: IWAMA, G. K.; PICKERING, A. D.; SUMPTER, J. P.; SCHRECK, C. B. (Ed.). Fish stress and health in aquaculture. Cambridge, Inglaterra: Cambridge University Press, 1997. p. 35-71. (Society for Experimental Biology Seminar Series, 62). 
WEDEMEYER, G. A.; McLEAY, D. J. Methods for determining the tolerance of fishes to environmental stressors. In: PICKERING, A. D. (Ed.). Stress and fish. London: Academic, 1981. p. 247-275.

WOOD, C. M.; WILSON, R. W.; GONZALEZ, R. J.; PATRICK, M. L.; BERGMAN, H. L.; NARAHARA, A.;
VAL, A. L. Responses of an Amazonian teleost, the tambaqui (Colossoma macropomum), to low $\mathrm{pH}$ in extremely soft water. Physiological Zoology, Chicago, v. 71, n. 6, p. 658-670, 1998.

WURTS, W. A. Using salt to reduce handling stress in channel catfish. World Aquaculture, Baton Rouge, v. 26, p. 80-81, 1995. 